\title{
Comparison of radiographic changes in rectangular curved short stem with thin versus thick porous coating for cementless total hip arthroplasty: a retrospective study with a propensity score matching
}

Yutaro Munakata, Yujiro Kuramitsu, Yutaka Usui and Ken Okazaki ${ }^{*}$

\begin{abstract}
Background: Radiographic changes that appear relatively quickly after fixation of cementless stem in total hip arthroplasty (THA) vary depending on the stem design and fixation style. The present study compared radiographic changes between two types of rectangular curved short stems of similar shape.

Methods: This retrospective study included 118 hips that underwent primary cementless THA with an anterolateral supine approach using a rectangular, curved, short stem performed by the same surgeon between June 2015 and June 2019. Among the examined hips, 39 had a thicker porous coating stem (thicker group) and 66 had a thinner porous coating and reduced tip stem (thinner group) and at least 12-month follow-up. Radiographs taken during the final course observation were assessed. Propensity score matching was performed based on demographic data and comparisons were made using pairs of 25 hips each. Statistical analysis was performed using chi-square test and $p$ values $\leq 0.05$ indicated statistical significance.
\end{abstract}

Results: The pattern of the radiolucent lines showed a significant difference after matching $(p=0.0044)$. A "proximal and distal" pattern was most common in the thicker group and a "distal only" pattern was most common in the thinner group. There was notable and significant difference in cortical hypertrophy in the thicker group after matching $(p=0.024)$.

Conclusions: Although the two short stems were similar shapes, the short-term radiographic changes were different. The thinner group showed fewer radiographic changes than the thicker group, making it a more "silent" stem.

Keywords: Short stems, Radiologic evaluation, Propensity score matched analysis

* Correspondence: okazaki.ken@twmu.ac.jp

Department of Orthopaedic Surgery, Tokyo Women's Medical University, 8-1,

Kawada-cho, Shinjuku-ku, Tokyo 162-8666, Japan

(c) The Author(s). 2021 Open Access This article is licensed under a Creative Commons Attribution 4.0 International License, which permits use, sharing, adaptation, distribution and reproduction in any medium or format, as long as you give appropriate credit to the original author(s) and the source, provide a link to the Creative Commons licence, and indicate if changes were made. The images or other third party material in this article are included in the article's Creative Commons licence, unless indicated otherwise in a credit line to the material. If material is not included in the article's Creative Commons licence and your intended use is not permitted by statutory regulation or exceeds the permitted use, you will need to obtain permission directly from the copyright holder. To view a copy of this licence, visit http://creativecommons.org/licenses/by/4.0/ The Creative Commons Public Domain Dedication waiver (http://creativecommons.org/publicdomain/zero/1.0/) applies to the data made available in this article, unless otherwise stated in a credit line to the data. 


\section{Background}

Total hip arthroplasty (THA), hailed as "the operation of the century," was the most successful surgery in the last century [1]. During its development, a "short stem" was designed to obtain more physiological load transfer with a less invasive procedure to preserve bone and soft tissue [2]. Recent comparisons with standard stems have reported that short stems are considered equivalent to standard stems in clinical assessment and imaging over short periods of time [3, 4]. Koyano et al. examined 36 cases of simultaneous bilateral THA, where a short stem was used on one side and a standard stem was used on the other, over an average of 9.2 years. Their radiological findings suggested that the short stem transferred the load more proximally than the standard stem, while no difference was observed in the hip score [5]. Yu et al. reported no difference in clinical assessment and imaging in 55 hips with short stem and 58 hips with standard stem, even for patients aged $\geq 70$ years, over short-term (3-4 years), and thigh pain and intraoperative fractures were less common with the short stem [6]. On the other hand, in an Italian regional registry, use of a short stem in the "standard osteotomy" group showed significantly more revisions due to pain compared with other groups [7]. According to the 2018 report by the Australian Orthopaedic Association National Joint Registry, loosening and revision rates were higher in mini stems than in conventional stems [8]. However, there were improvements in the 2020 report [9]. Therefore, controversy still exists regarding the radiological stability of short stems. One cause is that various design concepts are included within implants that are classified as short and mini stems. Furthermore, there may be differences among systems with similar shapes in terms of the surface finishing, such as the porous coating that may influence the postoperative radiographic changes.

Rectangular curved short stems comprise a short stem with a rectangular (trapezoidal) cross-section [10], similar to the Zweymüller type [11] with smooth curves. An example of this type of stem is the Fitmore (ZIMMER BIOMET, Warsaw, IN, USA, Fig. 1a), which has been reported to have stable short-term and midterm performance [4, 12-15], but the occurrence of cortical hypertrophy is reported to be common (2971\%) [12-16]. Meanwhile, there is a limited number of reports regarding the performance of the Minima (LIMA Corporate, San Daniele Del Friuli, Udine, Italy, Fig. 1b), $[17,18]$ rectangular curved short stem, and clinical results and radiographic changes have not been adequately examined. Although these systems have a similar shape, they differ in the shape of the tip, thickness, and extent of the porous coating. The Fitmore stem has a thicker porous coating than the Minima stem. Radiographical changes may develop relatively

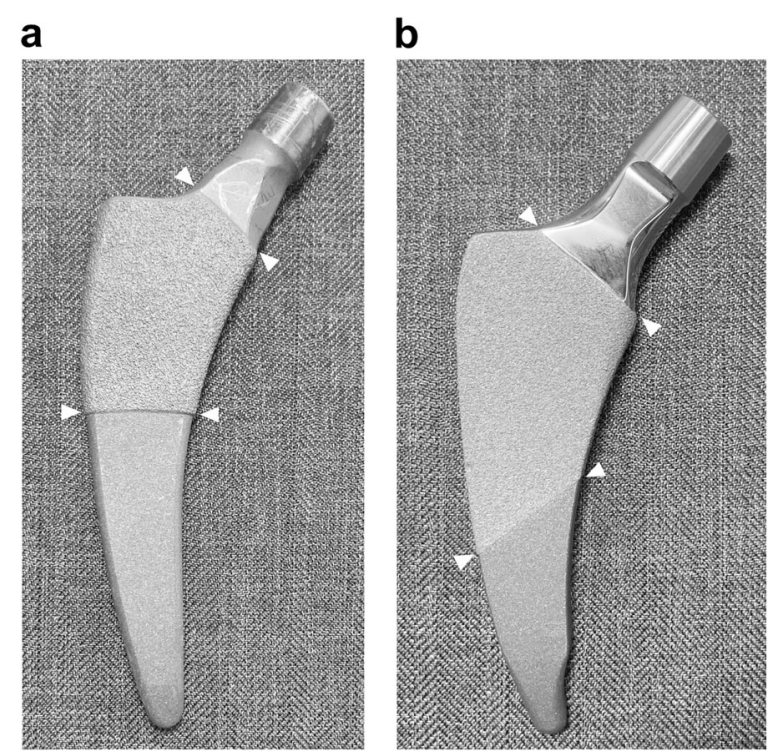

Fig. 1 Appearance of both the groups is depicted. a Appearance of the thicker group. It has thicker $(0.4 \mathrm{~mm})$, symmetrical porous coating and stem tip is not reduced. Arrows indicate the area of the porous coating. $\mathbf{b}$ Appearance of the thinner group. It has thinner $(0.2 \mathrm{~mm})$, asymmetrical porous coating and stem tip is reduced. Arrows indicate the area of the porous coating

quickly following surgery. They are often related to stem fixation and may be associated with clinical outcomes, such as the need for early revision $[19,20]$.

The present study aimed to elucidate the differences in radiographic changes that appear shortly after surgery using two types of rectangular curved short stems placed with the same surgical method by the same surgeon. We performed a propensity score-matched analysis to make patient background uniform using factors that likely have an impact on the result, including gender, age, body mass index, follow-up period, and morphology of the femoral canal [21].

\section{Methods}

The present study was reviewed by the ethics committee of our facility (approval number 5280, August 13, 2019). Informed consent was obtained using an opt-out form on the center's website. All data were handled according to the ethical standards of the Declaration of Helsinki.

The study included 124 hips that consecutively underwent primary cementless THA, which was performed with an anterolateral supine approach using a rectangular curved short stem by the same surgeon between June 2015 and June 2019. Preoperative diagnoses were hip dysplasia, avascular necrosis of the femoral head, rapidly destructive coxopathy, primary osteoarthritis of the hip, femoral neck fracture, rheumatoid arthritis, post-traumatic arthritis, and septic arthritis. The Fitmore and Minima stems were used and were selected by the surgeon based on the medial 
curvature of the proximal femur and lateral offset in preoperative two-dimensional templating. Fitmore has a thicker porous coating, while Minima has a thinner coating. Exclusion criteria were cases that underwent osteosynthesis for hip fracture, existing inflammatory disease, postoperative periprosthetic fracture, and cases that required additional surgery due to infection or problem with the cup side. Totals of 42 hips with Fitmore and 76 hips with Minima were included in the study. Among these, 13 hips could not be followed up for 12 months; thus, ultimately the study included 39 hips with Fitmore (thicker group) and 66 hips with Minima (thinner group). The follow-up rate was $89.0 \%$. We collected data for age, gender, and body mass index for these cases from medical records. In order to assess the femoral canal morphology, we measured the canal flare index [22] from preoperative anteroposterior radiograph in a supine position.

\section{Clinical assessment}

We measured the Japanese Orthopaedic Association (JOA) hip score prior to surgery and at the time of final course observation for evaluation. JOA hip score is an objective clinical score consisting of four subcategories, including pain around the hip joint, range of motion, walking ability, and activities of daily living, validated in patients with hip osteoarthritis [23].

\section{Radiological assessment}

We used an anteroposterior radiograph in a supine position and Lauenstein images immediately after surgery and at the time of the final course observation to evaluate radiographic changes in the areas around the stem after surgery. For stress shielding, we modified the Engh Grading Scale [24] and made an evaluation based on anteroposterior radiograph in the supine position only. No clear bone resorption was defined as "nothing," calcar rounding was defined as first degree, bone resorption advanced to the bottom of the lesser trochanter was defined as second degree, bone resorption advanced beyond the lesser trochanter was defined as third degree, and bone resorption advanced to the stem tip was defined as fourth degree. Since no cases were classed as fourth degree, we only assessed to third degree (Fig. 2). The radiolucent lines were assessed in two directions (anteroposterior radiograph in the supine position and Lauenstein imaging) and findings $\geq 1 \mathrm{~mm}$ was considered "present." Anterior and lateral modified Gruen zones [17, 25] (Fig. 3) 1, 7, 8, and 14 were defined as "proximal," and 3, 4, 5, 10, 11, and 12 were defined "distal," and then classified as either "nothing," "proximal only" (more proximal than the top of the lesser trochanter), "distal only," or "proximal and distal." No cases showed a radiolucent line that straddled the middle part extending from proximal to distal throughout the whole length. Cortical hypertrophy was also examined in the same two directions, and the number of confirmed modified Gruen zones [17, 25] (Fig. 3) was evaluated. Stem subsidence was only evaluated on the anteroposterior radiograph in the supine position, and subsidence of $\geq 3 \mathrm{~mm}$ was considered significant.

Interobserver and intraobserver reproducibility was assessed using two examiners to evaluate radiograph twice in a blinded manner in 10 randomly selected patients.

\section{Statistical analysis}

Statistical analysis of the interobserver and intraobserver reproducibility was performed using Gauge R \& R analysis. Student's $t$ test was used for continuous variables with normal distributions. Chi-square test was used for categorical variables. All $p$ values $\leq 0.05$ were considered statistically significant. Propensity score matching was performed to adjust covariates including gender, age, body mass index, follow-up period, and canal flare index with a caliper width of 0.2 as a logit transformation [26].

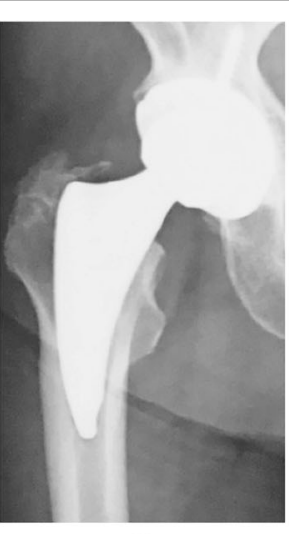

significant

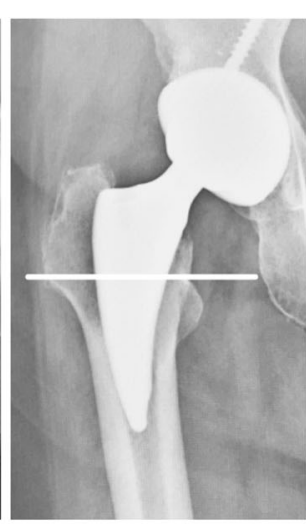

$1^{\circ}$ Calcar rounding

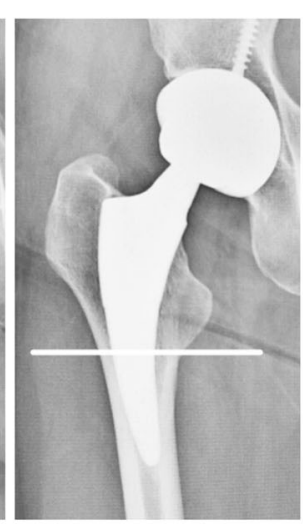

$2^{\circ}$

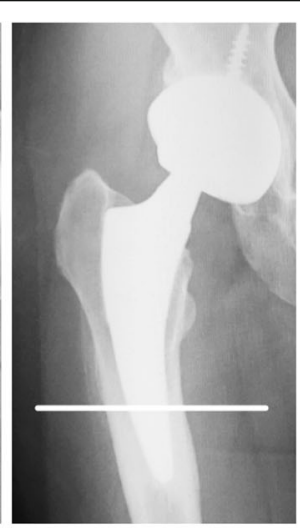

$3^{\circ}$

Fig. 2 Stress shielding assessment. LT, lesser trochanter 


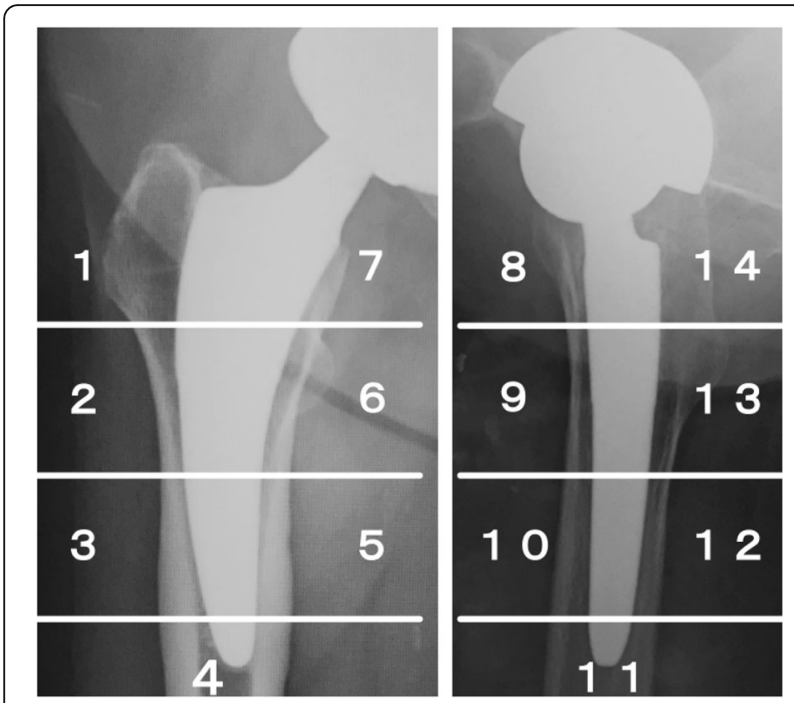

Fig. 3 Gruen zones in anteroposterior and lateral radiograph. According to this zoning, radiolucent lines and cortical hypertrophy were evaluated

Demographic data, JOA hip score, and radiographic changes were compared between the groups before and after matching. JMP Pro 13.0.0 (SAS, Cary, NC, USA) was used for statistical analyses.

\section{Results}

Results of the Gauge R \& R analysis for evaluating interobserver and intraobserver reproducibility showed $80 \%$ consistency and a Kappa value of 0.7 for stress shielding, $100 \%$ consistency and a Kappa value of 1 for radiolucent lines, $90 \%$ consistency and a Kappa value of 0.84 for cortical hypertrophy, and 100\% consistency and a Kappa value of 1 for stem subsidence. Reproducibility was acceptable in all the assessments.

The demographic data before and after matching is shown in Table 1. Before matching, there was significant

Table 1 Demographic data before and after propensity score matching. Data represent mean (standard deviation)

\begin{tabular}{llll}
\hline & Thicker group & Thinner group & $\boldsymbol{p}$ value \\
\hline Before matching & $n=39$ & $n=66$ & \\
Gender (male to female) & $11: 28$ & $8: 58$ & 0.064 \\
Age, years & $64.9(13.0)$ & $71.4(10.1)$ & 0.0053 \\
Body mass index, kg/m² & $23.0(4.5)$ & $23.9(5.0)$ & 0.36 \\
Follow-up, months & $33.4(11.5)$ & $23.3(11.3)$ & $<0.0001$ \\
Canal flare index & $3.4(0.5)$ & $3.5(0.4)$ & 0.18 \\
After matching & $n=25$ & $n=25$ & \\
Gender (male to female) & $4: 21$ & $3: 22$ & 1 \\
Age, years & $70.5(12.0)$ & $69.3(10.4)$ & 0.71 \\
Body mass index, kg/m² & $22.0(4.4)$ & $22.0(4.6)$ & 0.96 \\
Follow-up, months & $31.6(10.8)$ & $30.2(12.2)$ & 0.68 \\
Canal flare index & $3.4(0.55)$ & $3.4(0.46)$ & 0.76 \\
\hline
\end{tabular}

difference in gender, age, and follow-up between the groups, but these differences disappeared after matching.

JOA hip scores are shown in Table 2 as clinical results. Both groups showed notable improvements at the time of final course observation, but there was no significant difference between the groups before or after the matching.

The pre-matching and post-matching results for radiographic changes are shown in Table 3. Stress shielding showed a significant difference $(p=0.015)$ before matching, but this disappeared after matching ( $p$ $=0.26$ ). However, second degree was most common in thicker group (9 hips) and first degree was most common in thinner group (14 hips). The patterns of radiolucent lines showed a significant difference before matching $(p<0.0001)$ and after matching $(p=0.0044)$. In the thicker group, "proximal and distal" was most common and was seen in 10 hips (Fig. 4), while "distal only" was most common in the thinner group and was seen in 15 hips (Fig. 5). Cortical hypertrophy showed a significant $(p=0.013)$ difference before matching and after matching $(p=0.024)$ and was confirmed in $20 \%$ of the thicker group but not in the thinner group. Stem subsidence $(\geq 3 \mathrm{~mm})$ did not show a significant difference before $(p=0.55)$ or after matching $(p=1.0)$.

\section{Discussion}

The most important finding of the present study was the difference in radiographic changes shortly after surgery between the two models of similar shaped stems that were both rectangular, curved, and short but had a different thickness of porous coating. "Proximal and distal" was the most common pattern of radiolucent lines in the thicker group, whereas cortical hypertrophy was also commonly observed. In contrast, "distal only" was the most common pattern of radiolucent lines for the thinner group around the reduced tip. Cortical hypertrophy was significantly less common in the thinner group. Despite the stems having similar shapes, minor differences in the design led to a difference in radiographic changes. There was no difference in terms of short-term clinical results of these two stems.

Table 2 JOA hip score results. Data represent mean (standard deviation)

\begin{tabular}{llll}
\hline & Thicker group & Thinner group & $\boldsymbol{p}$ value \\
\hline Before matching & $n=39$ & $n=66$ & \\
Pre-surgical & $31.7(22.7)$ & $33.6(16.7)$ & 0.61 \\
At the final follow-up & $90.8(8.0)$ & $89.5(7.4)$ & 0.39 \\
After matching & $n=25$ & $n=25$ & \\
Pre-surgical & $27.0(19.5)$ & $37.4(20.8)$ & 0.07 \\
At the final follow-up & $90.0(7.2)$ & $91.4(6.4)$ & 0.46 \\
\hline
\end{tabular}


Table 3 Results of radiographic changes before and after matching

\begin{tabular}{|c|c|c|c|c|c|c|}
\hline & Before matchin & & & After matching & & \\
\hline & $\begin{array}{l}\text { Thicker group } \\
(n=39)\end{array}$ & $\begin{array}{l}\text { Thinner group } \\
(n=66)\end{array}$ & $\overline{p \text { value }}$ & $\begin{array}{l}\text { Thicker group } \\
(n=25)\end{array}$ & $\begin{array}{l}\text { Thinner group } \\
(n=25)\end{array}$ & $p$ value \\
\hline Stress shielding & & & 0.015 & & & 0.26 \\
\hline Nothing & 4 & 10 & & 2 & 2 & \\
\hline 1st degree & 16 & 40 & & 8 & 14 & \\
\hline 2nd degree & 11 & 14 & & 9 & 7 & \\
\hline 3rd degree & 8 & 2 & & 6 & 2 & \\
\hline Radiolucent line & & & $<0.0001$ & & & 0.0044 \\
\hline Nothing & 10 & 19 & & 8 & 6 & \\
\hline Proximal only & 11 & 3 & & 3 & 2 & \\
\hline Distal only & 4 & 39 & & 4 & 15 & \\
\hline Proximal and distal & 14 & 5 & & 10 & 2 & \\
\hline Cortical hypertrophy & & & 0.013 & & & 0.024 \\
\hline Nothing & 29 & 62 & & 20 & 25 & \\
\hline 1 zone & 5 & 3 & & 1 & 0 & \\
\hline$\geq 2$ zones & 5 & 1 & & 4 & 0 & \\
\hline Stem subsidence & & & 0.29 & & & 1 \\
\hline Nothing or $<2 \mathrm{~mm}$ & 37 & 65 & & 24 & 24 & \\
\hline$\geq 3 \mathrm{~mm}$ & 2 & 1 & & 1 & 1 & \\
\hline
\end{tabular}

Short stem design varies greatly and there are many classifications. Feyen et al. defined standard and short stems based on stem length [27]. McTighe et al. proposed a classification based on the site of fixation and stem design [28]. Khanuja et al. classified cementless standard stems based on the style of fixation and load transfer [10]. Falez et al. proposed classification based on differences in the femoral neck resection height [29].

omez-Garćia et al. proposed to summarize existing classifications for coding [30]. As such, there are various

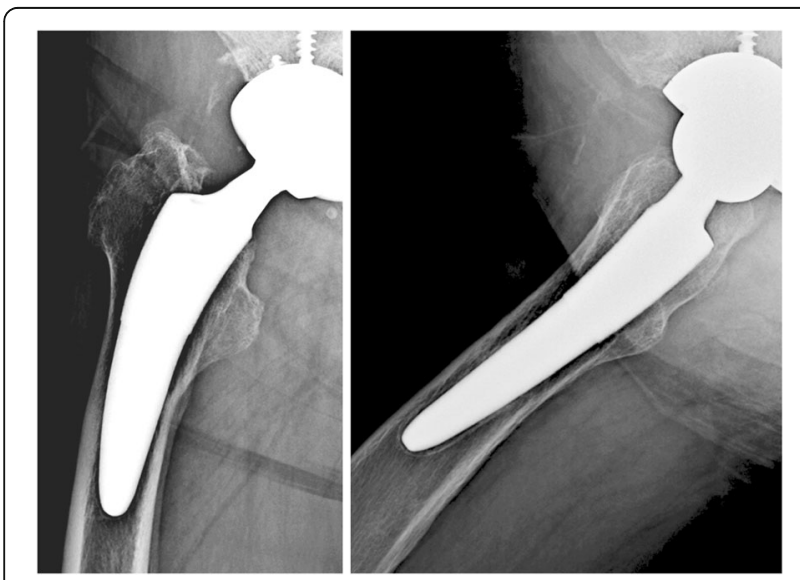

Fig. 4 Example of "proximal and distal" pattern. It was the most common pattern for radiolucent lines in the thicker group. Gruen zones $1,3,4,8,10,11$, and 12 showed radiolucent lines with widths of $1-2 \mathrm{~mm}$ classifications and no consensus [2]. However, the two stems used in the present study would be classified in the same group using any of these classifications: class IIIb, short stem with "standard" osteotomy in the classification by Feyen [27]; class 3A or 3B, short metaphyseal stabilized stems in the classification by McTighe [28];

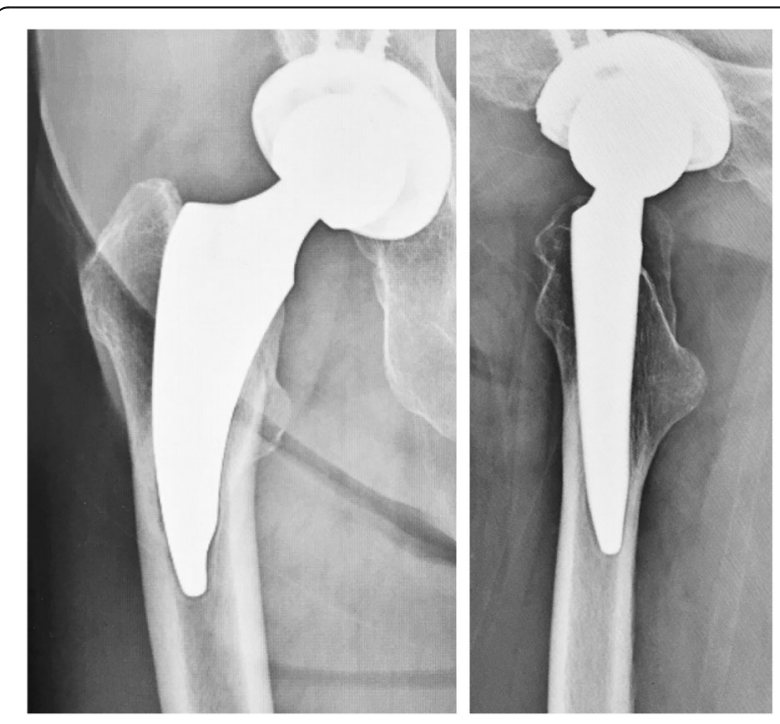

Fig. 5 Example of "distal only" pattern, the most extensive case in the thinner group. It was the most common pattern for radiolucent lines in the thinner group. Gruen zone 3, 4, 5, 10, 11, and 12 present radiolucent lines with the width of $1-2 \mathrm{~mm}$ 
type 4, shortened tapered conventional stem in the classification by Khanuja [10]; trochanter sparing in the classification by Falez [29]; and type C (cervicometaphyseal with proximal diaphyseal fixation) family, short, curved, without collar ("banana") in the classification by omez-Garćia [30]. Despite this, the present study confirmed significant differences in radiographic changes over a short-term follow-up.

There are several reports of different radiographic changes for similar stem design. Wick et al. compared the second and third generation Zweymüller type stems, Alloclassic, and SL-Plus [31], and reported that minor differences in manufacturing, alloy materials, and morphology led to changes in the load and rotational stress on the base. There could even be different bone responses based on minor difference in elements among rectangular curved short stems that are classified in the same group. Differences in the stems used in this study include distal tip shape, sagittal taper angle, extent and thickness of porous coating, and neck surface finish (Fig. 1a, b). These differences are confirmed in the manufacturer catalogs [32,33] or measured using enlarging digital template of ZedHip (Lexi, Toshima-ku, Tokyo, Japan), which is a three-dimensional preoperative planning tool. These differences may be associated with differences in the patterns of radiolucent lines and cortical hypertrophy where significant differences were observed between the two groups.

Radiolucent lines (reactive lines) that appear and advance over a wide area around the cementless stem with movements in the stem may be a sign of loosening $[19,20,34]$. However, radiolucent lines that are about 1-2 $\mathrm{mm}$ in width near the Zweymüller type stem, which is a rectangular straight standard-length stem, have no impact on the clinical result and are not a sign of loosening for a distally fixed stem [11]. Zweymüller, who developed the stem, hypothesized two causes of radiolucent lines: differences in the volume between the bone resected during the stage of rasping (preparation) and the actual stem, and the difference in rigidity with the metal stem when the proximal base bone (trochanter) is in a motion caused by the muscle tension if a stem is distally fixed under the trochanter [11]. With regard to the stem of thicker group in the present study, Maier et al. reported that radiolucent lines were confirmed at 11\% each in Gruen zones 1 and 7 (proximal), $2 \%$ in zone 2 and $3 \%$ in zone 6 (middle), and $2 \%$ in zone 3 and $9 \%$ in zone 5 (distal) during a follow-up of $\geq 2$ years [13]. Thalmann et al. reported that radiolucent lines were confirmed at $51 \%$ in the anteroposterior image and 35\% in the axial image in all zones other than the Gruen zone 2 during the first year, which decreased to $20 \%$ in the anteroposterior image and $9 \%$ in the axial image 5 years later [14]. In the present study, "proximal and distal" was the most common pattern of radiolucent lines for the thicker group $(35.9 \%$ before matching and $40 \%$ after matching). On the other hand, Drosos et al. reported clinical results for the stem used in the thinner group, where radiolucent lines were confirmed at 3.3\% (two hips) with distal pattern in Gruen zones 3,4 , and 5 [17]. In the present study, "distal only" was the most common pattern of radiolucent lines in the thinner group (59\% before matching and 60\% after matching). The reason for higher incidences compared with previous reports may be the advanced age of patients (average of 71 years prior to matching and 69 years after matching), leading to possible issues with bone quality. The tip of the stem of the thinner group was reduced, but the rasp tip was not. Radiolucent lines at the tip of the stem in the thinner group were caused by differences in the volume between the rasp and the actual stem (Fig. 6), as reported by Zweymüller [11].

There are many reports on cortical hypertrophy with the stem of the thicker group. Loppini et al. performed a systematic review of nine types of short stems with different site of fixation, morphology, and neck resection height [35]. There was no report of cortical hypertrophy exceeding 10\% for any stem other than the stem of the thicker group. Yan et al. evaluated changes in periprosthetic bone mineral density (BMD) in four types of short stems and two types of standard stems within 1 year of an operation in a systematic review [36]. BMD increased both inside and outside the distal area in Gruen zones 3 and 5 only for the stem of the thicker group, indicating possible distal fixation. One reason for cortical hypertrophy being common in this stem includes distal fixation [12, 13], nonideal press-fit and subsidence leading to distal two-point fixation [14], and increased lateral

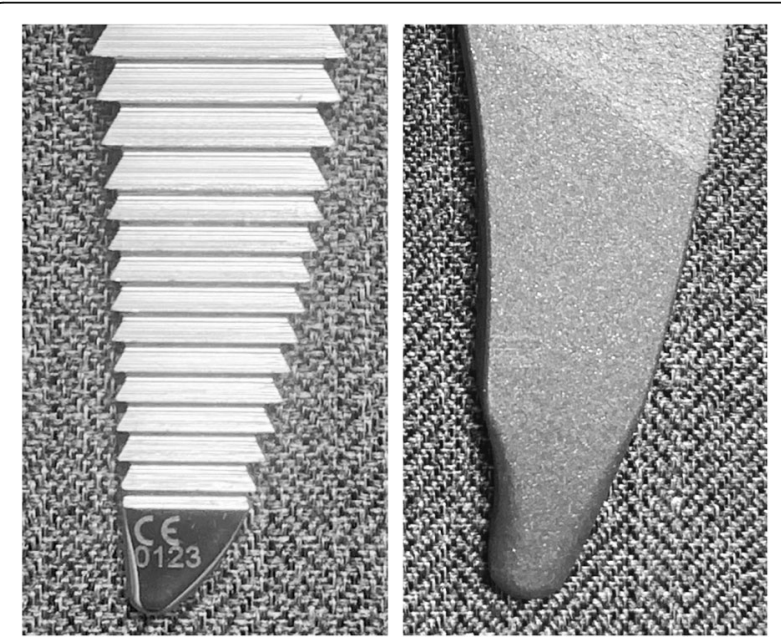

Fig. 6 Geometrical differences in thinner group rasp (left) and actual implant of the same size (right). The actual implant showed a reduced tip but not rasp 
offset [15]. Pepke et al. argued that although fixation is proximal, the high rigidity of mediolateral bending stress could lead to cortical hypertrophy [16]. The present study included cases with coexisting distal radiolucent lines and cortical hypertrophy (Fig. 7); thus, distal fixation is not necessarily the cause of cortical hypertrophy. Regarding the stem used in the thinner group, Drosos et al. observed $6.6 \%$ cortical hypertrophy in a year-long observation of 61 hips in the thinner group [17]. In the present study, $25.6 \%$ cortical hypertrophy was confirmed before matching and $20 \%$ after matching in the thicker group. In contrast, $6.1 \%$ was confirmed before matching and $0 \%$ after matching in the thinner group. These stems have similar shape and use the same material (titanium alloy); thus, the rigidity of the stem itself was assumed to be similar.

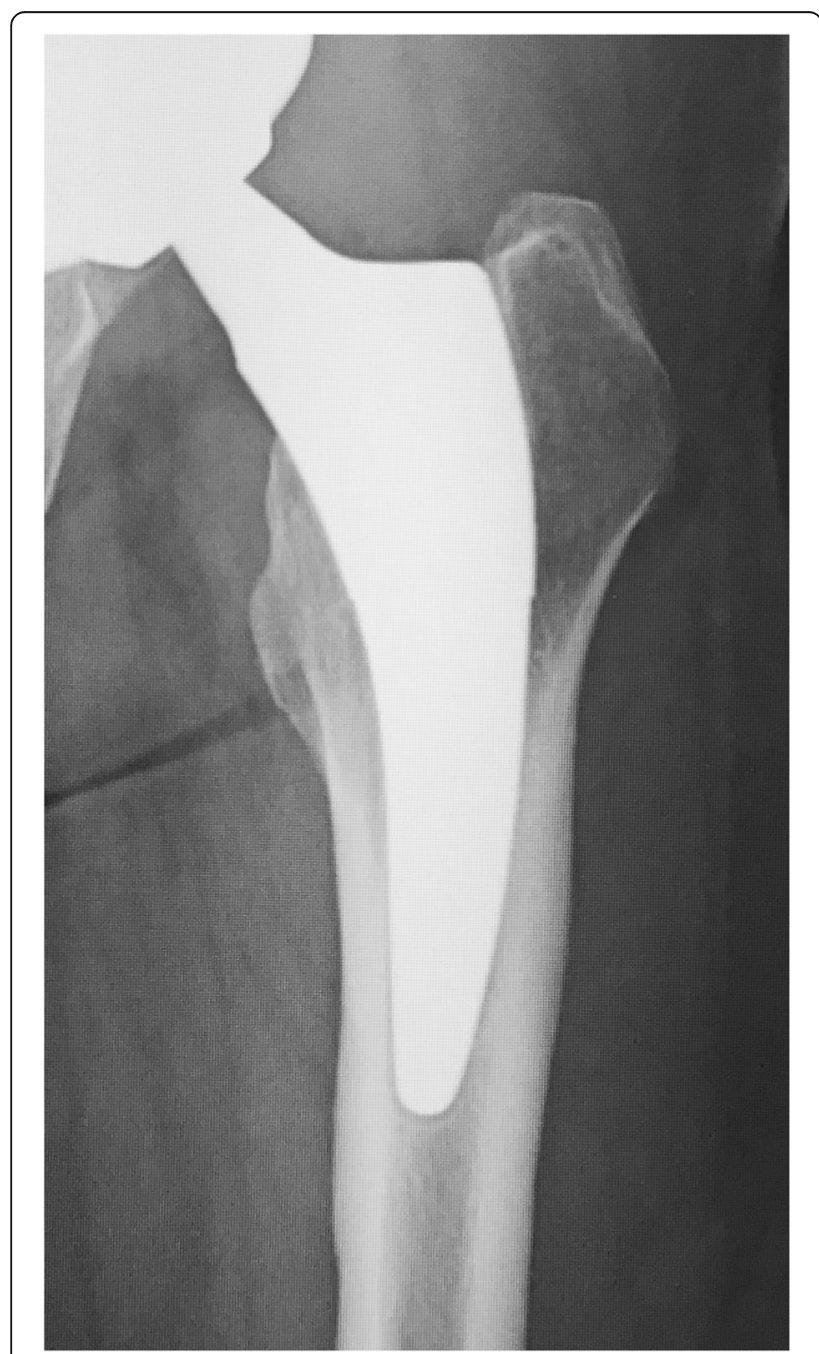

Fig. 7 Anteroposterior radiograph at 3 years after surgery in the thicker group. Radiolucent lines were confirmed in zones 3, 4, and 5 . Cortical hypertrophy was also confirmed in zones 3, 4, and 5
Differences in the thickness of the porous coating of these stems may lead to differences in the patterns of the radiolucent line and cortical hypertrophy. If stems have the same shape, the thicker coating requires more impaction stress to reach the same depth [37]. Stems with a thicker coating require more impaction stress for appropriate installation, and the press-fit force is focused on the distal part of the coating (around the middle part of the stem). Thus, the load stress is focused on the middle part of the stem, and micromotions are generated in the proximal and distal parts if the stem is only fixed at the middle part due to differences in the rigidity between the stem and bone [11]. This may explain why "proximal and distal" is the most common pattern of radiolucent lines in the thicker group. As the stem comes into contact with the cortex in Gruen zones 3 and 5 (distal part), the stress leads to increase in cortical hypertrophy. However, since standard stems with good long-term results also present various radiographic changes [38], it remains unclear how such imaging findings in rectangular curved short stems change over a long period of time, impacting the clinical results. There have been reports that radiographic changes may be linked to early revision [19, 20]; thus, although the short-term clinical results showed no difference in the present study, these radiographic changes would be of clinical concern.

The present study has several limitations. First, acetabular components and bearing couples were not uniform, even after matching. In the present cases, we used BIOLOX delta (CERAMTEC GmbH, Plochingen, Germany) as the femoral head, but the diameter of the femoral head varied between $\phi 28$ and $\phi 40 \mathrm{~mm}$. Two types of acetabular component and liner were used in the thicker group, whereas four types were used in the thinner group. However, in terms of differences in the material of the bearing couples and the femoral head diameter, friction can be ignored for the short period (within 5 years) that we examined in the present study [39-42]. Second, we did not make a comparison of patient-reported outcome measures (PROMs) [43]. While JOA hip score is not a PROM, many reports have argued that stress shielding, radiolucent line, and cortical hypertrophy, which were imaged in the present study, are unrelated to clinical symptoms $[5,6,11-15,17,24]$. Furthermore, the follow-up period was short and the number of cases was small. It is important to observe how the changes seen in the present study would change over longer follow-up periods. One of the strengths of the present study was that it used the same approach performed by the same surgeon. Furthermore, a propensity score-matched analysis was performed and comparisons were made after eliminating demographic differences, including femoral canal morphology. 


\section{Conclusions}

Two similarly shaped rectangular curved short stems exhibited significant differences in radiographical changes over a short period of time compared with after equalizing demographic data including the femoral canal morphology through a propensity score-matched analysis. Even when the morphology is similar, minor differences in stem design, such as thickness of the porous coating, can lead to radiographic changes shortly after surgery. The most common pattern of radiolucent lines in the thicker group was proximal and distal, while it distal only was most common in the thinner group. The incidence of cortical hypertrophy was significantly higher in the thicker group, whereas the result was more "silent" for the thinner group.

\section{Abbreviations}

BMD: Bone mineral density; JOA: Japanese Orthopaedic Association; PROM: Patient-reported outcome measures; THA: Total hip arthroplasty

\section{Acknowledgements}

The authors thank Crimson Interactive Pvt. Ltd. (Ulatus)—www.ulatus.jp—for their assistance in manuscript translation and editing.

\section{Authors' contributions}

YM performed surgery on all patients, analyzed and interpreted the patient data in the study, and wrote the manuscript draft. YK and YU assisted as surgical assistants and helped in analyzing the radiographs. KO assisted in the analysis and interpretation of the data, developed the overall structure of this study, reviewed, and edited the manuscript. All authors have read and have approved the final manuscript.

\section{Funding}

The authors declare that they have received no funding for the present study.

\section{Availability of data and materials}

The datasets used and/or analyzed during the current study are available from the corresponding author on reasonable request.

\section{Declarations}

\section{Ethics approval and consent to participate}

This study was reviewed by the ethics committee of our facility (approval number 5280, August 13, 2019). Informed consent was obtained using an opt-out form on the center's website. All data were handled according to the ethical standards of the Declaration of Helsinki.

\section{Consent for publication}

All the authors have read and agreed to its content and are accountable for all aspects of the accuracy and integrity of the manuscript in accordance with ICMJE criteria and confirmed that the article is original, has not already been published in a journal, and is not currently under consideration by another journal.

All the authors agree with the publication in the Journal of Orthopaedic Surgery and Research under the BioMed Central Copyright and License Agreement.

\section{Competing interests}

The authors declare that they have no competing interests.
Received: 12 February 2021 Accepted: 5 April 2021

Published online: 13 April 2021

\section{References}

1. Learmonth ID, Young C, Rorabeck C. The operation of the century: total hip replacement. Lancet. 2007;370(9597):1508-19. https://doi.org/10.1016/S01406736(07)60457-7.

2. Kim JT, Yoo JJ. Implant design in cementless hip arthroplasty. Hip Pelvis. 2016;28(2):5-65. https://doi.org/10.5371/hp.2016.28.2.65.

3. Huo SC, Wang F, Dong LJ, Wei W, Zeng JQ, Huang HX, et al. Short-stem prostheses in primary total hip arthroplasty: a meta-analysis of randomized controlled trials. Medicine. 2016;95(43):e5215. https://doi.org/10.1097/MD. 0000000000005215.

4. Freitag $T$, Hein MA, Wernerus D, Reichel $H$, Bieger R. Bone remodelling after femoral short stem implantation in total hip arthroplasty: 1-year results from a randomized DEXA study. Arch Orthop Trauma Surg. 2016;136(1):125-30. https://doi.org/10.1007/s00402-015-2370-z.

5. Koyano G, Jinno T, Koga D, Yamauchi Y, Muneta T, Okawa A. Comparison of bone remodeling between an anatomic short stem and a straight stem in 1-stage bilateral total hip arthroplasty. J Arthroplasty. 2017;32(2):594-600. https://doi.org/10.1016/j.arth.2016.07.016.

6. Yu H, Liu H, Jia M, Hu Y, Zhang Y. A comparison of a short versus a conventional femoral cementless stem in total hip arthroplasty patients 70 years and older. J Orthop Surg Res. 2016;11(1):33. https://doi.org/10.1186/ s13018-016-0367-0.

7. Giardina F, Castagnini F, Stea S, Bordini B, Montalti M, Toni A. Short stem versus conventional stems in cementless total hip arthroplasty: a long-term registry study. J Arthroplasty. 2018;33(6):1794-9. https://doi.org/10.1016/j.a rth.2018.01.005.

8. Australian Orthopaedic Association National Joint Replacement Registry [Annual report]. Adelaide: Australian Orthopaedic Association; 2018. https://a oanjrr.sahmri.com/documents/10180/576950/Hip\%2C\%20Knee\%20\%26\%2 OShoulder\%20Arthroplasty. Accessed 27 Jan 2021.

9. Australian Orthopaedic Association National Joint Replacement Registry [Annual report]. Adelaide: Australian Orthopaedic Association; 2020. https://a oanjr.sahmri.com/documents/10180/689619/Hip\%2C+Knee+\%26+ Shoulder+Arthroplasty+New/6a07a3b8-8767-06cf-9069-d165dc9baca7. Accessed 27 Jan 2021

10. Khanuja HS, Banerjee S, Jain D, Pivec R, Mont MA. Short bone-conserving stems in cementless hip arthroplasty. J Bone Joint Surg Am. 2014;96(20): 1742-52. https://doi.org/10.2106/JBJS.M.00780.

11. Zweymüller KA, Schwarzinger UM, Steindl MS. Radiolucent lines and osteolysis along tapered straight cementless titanium hip stems - a comparison of 6-year and 10-year follow-up results in 95 patients. Acta Orthop. 2006;77(6):871-6. https://doi.org/10.1080/17453670610013150.

12. Gustke K. Short stems for total hip arthroplasty: initial experience with the Fitmore stem. J Bone Joint Surg Br. 2012;94;Suppl A:47-1.

13. Maier MW, Streit MR, Innmann MM, Krüger M, Nadorf J, Kretzer JP, et al. Cortical hypertrophy with a short, curved uncemented hip stem does not have any clinical impact during early follow-up. BMC Musculoskelet Disord. 2015;16(1):371. https://doi.org/10.1186/s12891-015-0830-9.

14. Thalmann C, Kempter P, Stoffel K, Ziswiler T, Frigg A. Prospective 5-year study with 96 short curved Fitmore ${ }^{\text {TM }}$ hip stems shows a high incidence of cortical hypertrophy with no clinical relevance. J Orthop Surg Res. 2019; 14(1):156. https://doi.org/10.1186/s13018-019-1174-1.

15. Innmann MM, Weishorn J, Bruckner T, Streit MR, Walker T, Gotterbarm T, et al. Fifty-six percent of proximal femoral cortical hypertrophies 6 to 10 years after total hip arthroplasty with a short cementless curved hip stem a cause for concern? BMC Musculoskelet Disord. 2019;20(1):261. https://doi. org/10.1186/s12891-019-2645-6.

16. Pepke W, Nadorf J, Ewerbeck V, Streit MR, Kinkel S, Gotterbarm T, et al. Primary stability of the Fitmore ${ }^{\circledast}$ stem: biomechanical comparison. Int Orthop. 2014;38(3):483-8. https://doi.org/10.1007/s00264-013-2138-4.

17. Drosos Gl, Tottas S, Kougioumtzis I, Tilkeridis K, Chatzipapas C, Ververidis A. Total hip replacement using MINIMA ${ }^{\oplus}$ short stem: a shortterm follow-up study. World J Orthop. 2020;11(4):232-42. https://doi. org/10.5312/wjo.v11.i4.232.

18. Tatani I, Megas P, Panagopoulos A, Diamantakos I, Nanopoulos P, Pantelakis S. Comparative analysis of the biomechanical behavior of two different design metaphyseal-fitting short stems using digital image 
correlation. Biomed Eng OnLine. 2020;19(1):65. https://doi.org/10.1186/ s12938-020-00806-y.

19. Stenicka S, Hanreich C, Babeluk R, Kubista B, Giurea A, Sigmund IK, et al. High revision rates of a cementless beta-titanium alloy stem with contamination-free roughened surface in primary total hip arthroplasty. J Clin Med. 2020;9(7):2138. https://doi.org/10.3390/jcm9072138.

20. Macheras GA, Lepetsos P, Galanakos SP, Papadakis SA, Poultsides LA, Karachalios TS. Early failure of an uncemented femoral stem, as compared to two other stems with similar design, following primary total hip arthroplasty performed with direct anterior approach. Hip Int 2020;1120700020940671:112070002094067. https://doi.org/10.1177/112 0700020940671

21. Austin PC. An introduction to propensity score methods for reducing the effects of confounding in observational studies. Multivariate Behav Res. 2011;46(3):399-424. https://doi.org/10.1080/00273171.2011.568786.

22. Noble PC, Alexander JW, Lindahl L, Yew DT, Granberry WM, Tullos HS. The anatomic basis of femoral component design. Clin Orthop Relat Res. 1988; 235:148-65.

23. Kuribayashi M, Takahashi KA, Fujioka M, Ueshima K, Inoue S, Kubo T. Reliability and validity of the Japanese Orthopaedic Association hip score. J Orthop Sci. 2010;15(4):452-8. https://doi.org/10.1007/s00776010-1490-0.

24. Engh CA, Bobyn JD, Glassman AH. Porous-coated hip replacement - the factors governing bone ingrowth, stress shielding, and clinical results. J Bone Joint Surg. 1987;69:45-55.

25. Gruen TA, McNeice GM, Amstutz HC. 'Modes of failure' of cemented stemtype femoral components. Clin Orthop Relat Res. 1979;141:7-17.

26. Austin PC. Optimal caliper widths for propensity-score matching when estimating differences in means and differences in proportions in observational studies. Pharm Stat. 2011;10(2):150-61. https://doi.org/10.1002/pst.433.

27. Feyen $\mathrm{H}$, Shimmin AJ. Is the length of the femoral component important in primary total hip replacement? Bone Joint J. 2014;96-B(4):442-8. https://doi. org/10.1302/0301-620X.96B4.33036.

28. McTighe T, Keggi J, Stulberg SD, Keppler L, Brazil D, McPherson EJ. Total hip stem classification system. JISRF Reconstr Rev. 2014;4.

29. Falez F, Casella F, Papalia M. Current concepts, classification and results in short stem hip arthroplasty. Orthopedics. 2015;38(3):S6-13. https://doi.org/1 0.3928/01477447-20150215-50.

30. Ǵomez-Garćia F, Fernández-Fairen M, Espinosa-Mendoza RL. A proposal for the study of cementless short-stem hip prostheses. Acta Orthop Méxicana. 2016;30:204-15.

31. Wick M, Lester DK. Radiological changes in second- and third-generation Zweymüller stems. J Bone Joint Surg. 2004;86:1108-1114.

32. Fitmore ${ }^{\oplus}$ Hip stem-product information. 06(01558). Zimmer. 2011. https:// www.zimmerbelgium.be/content/dam/zimmer-web/documents/en-GB/pdf/ medical-professionals/hip/Fitmore-Hip-Stem-Brochure-06.01558.012-10-2011. pdf. Accessed 27 Jan 2021.

33. Minima ${ }^{\oplus}$ hip replacement flyer. B-4502-83-020-1, Lima Corporate. 2015 https://limacorporate.com/repo/storage/325/file/B-4502-83-020-1_091500_ FLY_MINIMA_EN.pdf. Accessed 27 Jan 2021.

34. Engh CA, Massin P, Suthers KE. Roentgenographic assessment of the biologic fixation of porous-surfaced femoral components. Clin Orthop Relat Res. 1990;257:107-28.

35. Loppini M, Grappiolo G. Uncemented short stems in primary total hip arthroplasty: the state of the art. EFORT Open Rev. 2018;3(5):149-59. https:// doi.org/10.1302/2058-5241.3.170052.

36. Yan SG, Weber P, Steinbrück A, Hua X, Jansson V, Schmidutz F. Periprosthetic bone remodelling of short-stem total hip arthroplasty: a systematic review. Int Orthop. 2018;42(9):2077-86. https://doi.org/10.1007/ s00264-017-3691-z.

37. Miles B, Walter WL, Kolos E, Waters T, Appleyard R, Gillies RM, et al. A plasma-sprayed titanium proximal coating reduce the risk of periprosthetic femoral fracture in cementless hip arthroplasty. Bio Med Mater Eng. 2015; 25(3):267-78. https://doi.org/10.3233/BME-151279.

38. Rivière C, Grappiolo G, Engh CA Jr, Vidalain JP, Chen AF, Boehler N, et al. Long-term bone remodelling around "legendary" cementless femoral stems. EFFORT Open Rev. 2018;3(2):45-57. https://doi.org/10.13 02/2058-5241.3.170024.

39. AL-Hajjar M, Fisher J, Tipper JL, Williams S, Jennings LM. Wear of 36-mm BIOLOX delta ceramic-on-ceramic bearing in total hip replacements under edge loading conditions. Eng Med. 2013;227:535-42.
40. Boyer B, Neri T, Geringer J, Di lorio A, Philippot R, Farizon F. Long-term wear of dual mobility total hip replacement cups: explant study. Int Orthop. 2018; 42(1):41-7. https://doi.org/10.1007/s00264-017-3525-z.

41. Gaudiani MA, White PB, Ghazi N, Ranawat AS, Ranawat CS. Wear rates with large metal and ceramic heads on a second generation highly cross-linked polyethylene at mean 6-year follow-up. J Arthroplasty. 2018;33(2):590-4. https://doi.org/10.1016/j.arth.2017.09.006.

42. Galea VP, Connelly JW, Shareghi B, Kärrholm J, Sköldenberg O, Salemyr M, et al. Evaluation of in vivo wear of vitamin E-diffused highly crosslinked polyethylene at five years: a multicentre radiostereometric analysis study. Bone Joint J. 2018;100-B(12):1592-9. https://doi.org/10.1302/0301-620X.1 O0B12.BJJ-2018-0371.R1.

43. Siljander MP, McQuivey KS, Fahs AM, Galasso LA, Serdahely KJ, Karadsheh MS. Current trends in patient-reported outcome measures in total joint arthroplasty: a study of 4 major orthopaedic journals. J Arthroplasty. 2018; 33(11):3416-21. https://doi.org/10.1016/j.arth.2018.06.034.

\section{Publisher's Note}

Springer Nature remains neutral with regard to jurisdictional claims in published maps and institutional affiliations.
Ready to submit your research? Choose BMC and benefit from:

- fast, convenient online submission

- thorough peer review by experienced researchers in your field

- rapid publication on acceptance

- support for research data, including large and complex data types

- gold Open Access which fosters wider collaboration and increased citations

- maximum visibility for your research: over $100 \mathrm{M}$ website views per year

At $\mathrm{BMC}$, research is always in progress.

Learn more biomedcentral.com/submissions 